SCIENCE CHINA

Physics, Mechanics \& Astronomy

\title{
Signal-in-space accuracy for BeiDou navigation satellite system: Challenges and solutions
}

\author{
ShanShi Zhou*, Bin $\mathrm{Wu}^{*}$, XiaoGong $\mathrm{Hu}$, YueLing Cao, and Yang Yu \\ Shanghai Astronomical Observatory, Chinese Academy of Sciences, Shanghai 200030, China
}

Received September 28, 2016; accepted October 14, 2016; published online November 21, 2016

\begin{abstract}
Citation: $\quad$ S. S. Zhou, B. Wu, X. G. Hu, Y. L. Cao, and Y. Yu, Signal-in-space accuracy for BeiDou navigation satellite system: Challenges and solutions, Sci. China-Phys. Mech. Astron. 60, 019531 (2017), doi: 10.1007/s11433-016-0384-6
\end{abstract}

Service accuracy of global navigation satellite system (GNSS) is determined by satellite constellation, signal-in-space (SIS) accuracy and signal transmission errors. The State Council Information Office of the People's Republic of China issued China's BeiDou Navigation Satellite System (BDS) White Paper (www.beidou.gov.cn/fbh2016.html) on Jun. $16^{\text {th }} 2016$, which notes that, BDS takes a three-step development strategy, from domestic service, through Asia-Pacific regional service, to global service, and features mixed constellation of Geostationary Earth Orbit (GEO), Inclined Geostationary Earth Orbit (IGSO) and Medium Earth Orbit (MEO) satellites, three frequencies navigation signal, and combination of navigation and communication services. And those features make the development of space segment and ground segment more complicated than Global Positioning System (GPS) and the processing of SIS (or navigation message) different from GPS.

However, BDS SIS accuracy meets some new problems and challenges concerning the following three aspects. First, space segment of BDS is a constellation of GEO/IGSO/MEO satellites. The stationary character of GEO satellites makes satellite orbital elements and clock estimations highly correlated with each other, which decreases orbit and clock parameters accuracy. Besides, in the period of spring/autumn equinox, improper solar radiation model of GEO satellites may bring about high correlation between orbital elements

*Corresponding authors (ShanShi Zhou, email: sszhou@shao.ac.cn; Bin Wu, email: bwu@shao.ac.cn) and thus reduce orbit determination accuracy. Second, to provide SIS processing, ground segment of BDS adopts a regional tracking network, whose dataset can't cover all the orbit of IGSO/MEO satellites and therefore precise long term predictions of satellite orbit and clock have to be provided by ground segment. Finally, since multi-path effects are serious for GEO's pseudorange, broadcast parameters can't be estimated by conventional methods precisely, and accuracy and stability of navigation service will decrease. This paper discusses new data processing methods adopted by BDS to handle with these problems.

Different from other GNSS, BDS adopts two-way satellite time and frequency transfer (TWSTFT) method to realize both station to station and satellite to ground time synchronization. Precise satellite clock errors are calculated by two-way pseduorange measurements, and then the Beidou Time (BDT) standard kept by ground atomic clock sets is transferred to each BDS satellite. The principle of satellite to ground TWSTFT and the results of TWSTFT for BDS test satellite are described in ref. [1]. And results show that the random noise of TWSTFT clock observations is about $0.1 \mathrm{~ns}$, the system error less than $1 \mathrm{~ns}$ and the accuracy of $2 \mathrm{~h}$ clock prediction better than 3 ns. TWSTFT clock observations and multi-satellite orbit determination (MPOD) estimations are compared in ref. [2]. Results indicate that TWSTFT clock observations are less affected by orbital error and thus more suitable for BDS satellite clock prediction. Furthermore, ref. [2] notes that the comparison result of TWSTFT clock observations and MPOD clock estimations can also be used to monitor navigation system time difference between BDT 
and other GNSS, and to evaluate the performance of satellite atomic clock in-orbit. These works represent the innovative applications of TWSTFT in BDS. Results in ref. [2] show that the stability of BDS satellite clock is similar to GPS IIR in $86400 \mathrm{~s}$ interval. According to ref. [3], short-term stability of BDS satellite clock is similar to GPS IIF rubidium frequency standard (RFS).

GEO satellites are the core component of BDS constellation. In the early stage of BDS regional system, limitation of monitoring network distribution seriously affected accuracy of precise orbit determination (POD) and orbit prediction, especially for GEO satellites. To solve the problem, a modified POD method combining C-band two-way ranging and L-band down-link pseudorange and carrier phase observation is proposed as is discussed in ref. [4]. And results show that adopting $3 \mathrm{C}$-band and 4 L-band stations' 3-d observations, the root mean square (RMS) of satellite laser ranging (SLR) residual is less than $0.1 \mathrm{~m}$, orbital radial accuracy of GEO satellite orbit $2 \mathrm{~h}$ prediction about $0.5 \mathrm{~m}$ evaluated by SLR. With more satellites deployed especially IGSO and MEO, it's possible to adopt MPOD strategy using L-band pseudorange and phase measurements from regional tracking network. Satellite orbital and clock parameters processing by MPOD strategy is proposed, and an indirect method to evaluate MPOD accuracy is proposed in ref. [5]. For a $4 \mathrm{GEO}+5 \mathrm{IGSO}+2 \mathrm{MEO}$ constellation the error of $24 \mathrm{~h}$ orbit overlap in radial $(\mathrm{R})$, transverse $(\mathrm{T})$ and normal $(\mathrm{N})$ direction is about $0.2 \mathrm{~m} / 1.2 \mathrm{~m} / 0.6 \mathrm{~m}$, the accuracy of satellite clock estimations about $1.4 \mathrm{~ns}$. Ref. [6] notes that during the period of spring and autumn equinox, when the Sun is near the earth's equatorial plane while GEO satellites are in deep shadow, adoption of the traditional solar radiation pressure model may make GEO orbital elements highly correlated and then significantly decreases the orbit determination accuracy. To handle with this problem, a new orbit determination strategy adopting BDS TWSTFT satellite clock observations and pseudorange and phase observations is proposed in ref. [7]. And the results show that user equivalent ranging error (UERE) of GEO satellites in the autumn equinox period of 2012 can significantly be improved from 2.5 to $1.3 \mathrm{~m}$ and the probability of UERE better than $2.0 \mathrm{~m}$ can be improved from $50 \%$ to above $85 \%$. To decrease the effect of regional tracking network on orbit determination, an MPOD processing method for BDS satellite in non-equinox period is proposed in ref. [8] which develops the method discussed in ref. [7]. Satellite clock measurements from TWSTFT are fixed as known values, and only orbital elements are solved. Results show that UERE is reduced by $27.6 \%$ for GEO satellites and SLR residual exhibit by $30 \%$ for IGSOs. GNSS broadcast ephemerides are evaluated in ref. [9]. Compared with precise orbit, BDS broadcast ephemerides accuracy in radial is about $0.48 \mathrm{~m}$, clock error about $0.98 \mathrm{~m}$, which are less than GPS but better than Galileo. BDS navigation message is processed using regional tracking data. Meanwhile, Multi-GNSS Experiment (MGEX) set-upped by the International GNSS Service (IGS) carried out BDS observation. Adopting global Multi-GNSS observations, a two-step GPS-assisted BDS orbit determination method is proposed in ref. [10]. Results show that this method helps to strengthen the receiver clock and zenith tropospheric delays estimations, and then the accuracy of BDS orbit is improved.

Different from GPS, BDS regional system provides not only basic navigation service, but also satellite-based augmentation system (SBAS) service (www.beidou.gov.cn). Ref. [11] shows wide-area differential algorithm and designs a real-time multipath correct method to decrease the multi-path effect on GEO code measurements. And the accuracy of differential corrections with multi-path error removed and basic and SBAS positioning are analyzed in details. Results show that accuracy of dual-frequency and single-frequency positioning is improved remarkably to 5 and $6 \mathrm{~m}$ (RMS) by basic navigation service and to 3 and $4 \mathrm{~m}$ (RMS) by SBAS service. The differential corrections are able to improve SIS accuracy and positioning accuracy remarkably. It is proved that the differential information can reduce orbit and clock errors due to the long term prediction and thus improve SIS accuracy effectively, especially for MEO satellites. To mitigate the multipath effects on GNSS processing, multipath hemispherical map (MHM) and sidereal filtering (SF)/advanced SF (ASF) are proposed and compared in ref. [12]. Comparisons indicate that both MHM and ASF approaches perform well with residual variance reduction $(50 \%)$ for short span (next $5 \mathrm{~d}$ ). The ASF model is more suitable for high frequency multipath reduction, while the MHM model is easier to implement for real-time multipath mitigation.

Providing regional navigation service simultaneously, BDS takes a test mission of new-generation BDS satellites in which SIS accuracy is improved with new techniques, which is analyzed in ref. [13]. Results show that the clock prediction accuracy of new-generation BDS satellites is greatly improved thanks to the new precise Rubidium and Passive Hydrogen Maser of great stability equipped. The short-term prediction errors of IGSO and MEO satellites clock drop respectively from 0.65 to $0.3 \mathrm{~ns}$ and from 0.78 to $0.32 \mathrm{~ns}$, and mid-term prediction errors from 2.5 to $1.5 \mathrm{~ns}$. Inter-Satellite Links (ISL) with measurement and data communication capabilities is one of the most important designs for global BDS system. With ISL measurements, MEO satellites clock prediction error decreases from $3 \mathrm{~ns}$ to within $1 \mathrm{~ns}$ when the satellites go into the visual field again. With an approach of combining ISL and regional L-band code and phase measurements, orbit determination and prediction accuracy is also improved greatly, the radial of $24 \mathrm{~h}$ orbit overlap better than $0.1 \mathrm{~m}, 24 \mathrm{~h}$ prediction radial overlap better than $0.2 \mathrm{~m}$. All these results demonstrate that compared with regional system new-gener- 
ation BDS has greatly enhanced SIS accuracy. In addition to modification of ground processing algorithms relying on ISL and ground tracking measurements, satellite autonomous orbit determination and time synchronization using ISL ranging and communication are also tested on the new-generation BDS satellites. A centralized kinematics orbit determination method based on long term broadcast ephemeris is proposed in ref. [14]. This method boasts an advantage of effective processing without EOP supported. Adopting 60-d long term orbit prediction, autonomous navigation orbit determination user ranging error (URE) is better than $1 \mathrm{~m}$ and three-dimensional position accuracy is better than $3 \mathrm{~m}$. It provides the possibility of achieving on-board centralized autonomous navigation processing.

Similar to GPS, BDS provides ionospheric delay model parameters, Klobuchar- 8 and Klobuchar-14 updated every two hours as well as satellite orbit and clock prediction updated every hour. What's more, an improved BDS ionospheric model during solar storms is proposed in ref. [15] which adopts a new strategy that increases the prediction accuracy by $70 \%$ during both ionospheric positive and negative storm period. Besides ionospheric delay model parameters, differential code biases (DCBs) of BDS satellite are also provided in navigation message. An algorithm of estimating ionospheric spherical harmonics coefficients and systematic DCBs adopting global BDS/GPS dual-system observations is proposed in ref. [16]. Results indicate that the standard deviations (STD) of BDS DCBs are less than $0.4 \mathrm{~ns}$ in 23 months' period and they only require occasional estimation.

All the new technologies and new algorithm adopted in BDS are aiming at improving BDS SIS accuracy. The SIS accuracy, URE and User Differential Range Error (UDRE),

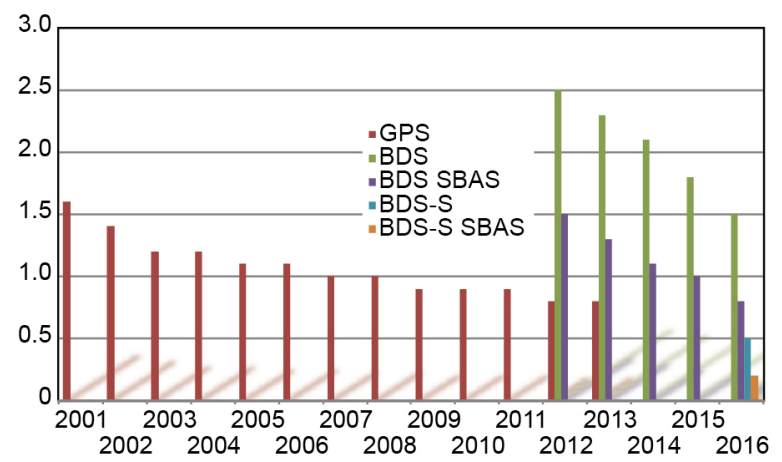

Figure 1 (Color online) GPS and BDS SIS accuracy time series. The red bars represent GPS URE (Data from www.gps.gov/systems/gps/performance/accuracy/), green and purple bars represent BDS regional system URE and UDRE (Data from www.beidou.org), and the blue and orange stand for BDS new-generation satellite URE and UDRE [13]. of BDS regional system is about 2.5 and $1.5 \mathrm{~m}$, and of the new-generation BDS it drops to 0.5 and $0.2 \mathrm{~m}$ respectively (see Figure 1). This paper focus on the new technology and methods adopted to enhance BDS SIS accuracy. Other experimental works such as the new navigation signal, new navigation message design, multi GNSS compatible and interoperability are also being carried out in refs. [17-19]. With all these promotions, BDS global system may provide higher SIS accuracy and better precise positioning, velocity and timing service.

This work was supported by the National Natural Science Foundation of China (Grant No. 41574029), the National Key Research Program of China "Collaborative Precision Positioning Project" (Grant No. 2016YFB0501900), and the Youth Innovation Promotion Association CAS (Grant No. 2016242).

1 L. Liu, G. F. Tang, C. H. Han, X. Shi, R. Guo, and L. F. Zhu, Sci. China-Phys. Mech. Astron. 58, 089502 (2015).

2 S. S. Zhou, X. G. Hu, L. Liu, R. Guo, L. F. Zhu, Z. Q. Chang, C. P. Tang, X. Q. Gong, R. Li, and Y. Yu, Sci. China-Phys. Mech. Astron. 59, 109511 (2016).

3 E. Griggs, E. R. Kursinski, and D. Akos, Radio Sci. 50, 813 (2015).

4 R. Guo, X. G. Hu, B. Tang, Y. Huang, L. Liu, L. C. Cheng, and F. He, Chin. Sci. Bull. 55, 687 (2010).

5 S. S. Zhou, X. G. Hu, J. H. Zhou, J. P. Chen, X. Q. Gong, C. P. Tang, B. Wu, L. Liu, F. He, X. J. Li, and H. L. Tan, in Accuracy Analyses of Precise Orbit Determination and Timing for COMPASS/Beidou-2 4GEO/5IGSO/4MEO Constellation: Proceedings of the China Satellite Navigation Conference (CSNC) 2013, edited by J. D. Sun,W. H. Jiao, H. T. Wu, and C. Shi (Springer, Berlin, Heidelberg, 2013), pp. 89-102.

6 X. J. Li, J. H. Zhou, X. G. Hu, L. Liu, R. Guo, and S. S. Zhou, Sci. China-Phys. Mech. Astron. 58, 089501 (2015).

7 F. He, S. S. Zhou, X. G. Hu, J. H. Zhou, L. Liu, R. Guo, X. J. Li, and S. Wu, Sci. China-Phys. Mech. Astron. 57, 1395 (2014).

8 C. Tang, X. Hu, S. Zhou, R. Guo, F. He, L. Liu, L. Zhu, X. Li, S. Wu, G. Zhao, Y. Yu, and Y. Cao, Adv. Space Res. 58, 1390 (2016).

9 O. Montenbruck, P. Steigenberger, and A. Hauschild, GPS Solut 19, 321 (2015).

10 Y. Lou, Y. Liu, C. Shi, B. Wang, X. Yao, and F. Zheng, GPS Solut 20, 259 (2016).

11 Y. L. Cao, X. G. Hu, B. Wu, S. S. Zhou, L. Liu, R. R. Su, Z. Q. Chang, F. He, and J. H. Zhou, Sci. China-Phys. Mech. Astron. 55, 1307 (2012).

12 D. Dong, M. Wang, W. Chen, Z. Zeng, L. Song, Q. Zhang, M. Cai, Y. Cheng, and J. Lv, J Geod 90, 255 (2016).

13 J. P. Chen, X. G. Hu, C. P. Tang, S. S. Zhou, R. Guo, J. Y. Pan, R. Li, and L. F. Zhu, Sci. Sin.-Phys. Mech. Astron. 46, 119502 (2016).

14 Y. Mao, X. G. Hu, X. Y. Song, Y. L. Chen, X. L. Jia, and X. B. Wu, Sci. Sin.-Phys. Mech. Astron. 45, 079512 (2015).

15 E. Q. Dong, Z. Q. Chang, X. J. Li, H. L. Tan, J. C. Fan, and X. L. Wu, Sci. Sin.-Phys. Mech. Astron. 45, 079504 (2015).

16 J. Xue, S. Song, and W. Zhu, J Geod 90, 309 (2016).

17 W. Xiao, W. Liu, and G. Sun, GPS Solut 20, 125 (2016).

18 J. Chen, J. Wang, Y. Zhang, S. Yang, Q. Chen, and X. Gong, Sensors 16, 1151 (2016).

19 N. Wang, Y. Yuan, Z. Li, O. Montenbruck, and B. Tan, J Geod 90, 209 (2016). 